EPJ Web of Conferences 38, 16002 (2012)

DOI: $10.1051 /$ epjconf/20123816002

(c) Owned by the authors, published by EDP Sciences, 2012

\title{
Exchange effects in composite-particle interaction
}

\author{
S. Yu. Igashov', Yu. M. Tchuvil'sky² \\ ${ }^{1}$ All-Russia Research Institute of Automatics, 127055, Moscow, Russia \\ ${ }^{2}$ Skobeltsyn Institute of Nuclear Physics, Lomonosov Moscow State University, 119991, Moscow, Russia
}

\begin{abstract}
Algebraic version of the orthogonality conditions model is developed by analogy with the algebraic version of the resonating group model. It is shown that all exchange terms excluding ones originated by the exchange kernel of the potential energy can be easily taken into account in the frame of the formalism of the version. The potential term as a whole is modelled phenomenologically. Both direct algebraic approach and the method basing on the solution of the integro-differential Schrödinger equation containing nonlocal terms related to the forbidden and the semi-forbidden states are proposed. This equation turns out to be preferable in studies of narrow resonances. It is demonstrated that decay width of a system into two-heavy-fragment channel is strongly affected by the nonlocal terms.
\end{abstract}

\section{Introduction}

The traditional view on the interaction of two particles is to consider it in terms of a local potential model independently of the structure of interacting particles. At the same time in the realistic approach the interaction of composite particles, i.e. particles consisting of some fermions-constituents, should be described in an approach which takes into account multi-nucleon structure of the two-body system as a whole.

If the internal states of the interacting particles are fixed, the resonating group model (RGM) [1,2] allows one to reduce the problem of description of their interaction to two-body one. However resulting two-body equation turns out to be not Schrödinger one because it contains exchange integral kernels in all it's terms.

The methods of reduction of the RGM equation to the Schrödinger equation with a Hermitian Hamiltonian are well known (see, for example, [3]). However even after these rearrangements the RGM is an overcomplicated approach which looks still far from habituated scheme of description of two-body interaction. Any pair of composite particles should be described individually. In fact the RGM remains a many-nucleon but not a twobody technique.

Another problem of the RGM is its inflexibility. In consequence of the incompleteness of the space of solutions inherent for the model, experimental observables are not well reproduced in the RGM sometimes. And, obviously, three-, four- (if it would be reasonable to use) etc. composite-particle problems in the RGM cannot be reduced to two- composite-particle one.
The goal of the present paper is to construct the method which, on the one hand, allows one to account for the property of fermion identity in the system of two composite particles (and thus the Pauli exclusion principle and various exchange effects caused by this identity) as much as possible and, on the other hand, provides essentially higher flexibility than the RGM.

The basic step on the way is to develop a method similar to the one presented in the paper [4]. An approximation of the RGM which makes it more or less similar to ordinary two-body approach is proposed there. Various alternatives of this approach have been developed up to now. These approximations differ by methods of manipulation with the exchange terms. They are known under the unified name orthogonality conditions model (OCM).

In this paper the algebraic version (AV) of OCM is presented. It is originated in analogy with the AV RGM proposed in the Refs. [5-7]. In addition the integrodifferential equation of the Schrödinger type with a Hermitian Hamiltonian containing nonlocal terms related to the forbidden by the Pauli principle and so-called "semi-forbidden" states is obtained. This "comeback" to the methods of the continuous mathematics turns out to be rather convenient in description of the widths of narrow resonances decaying into cluster-cluster channels.

For the heavy-ion interactions it is demonstrated by means of the algebraic version of the orthogonality conditions model that the values of the decay widths are strongly affected by the exchange effects. The pair of magic fragments ${ }^{16} \mathrm{O}+{ }^{16} \mathrm{O}$ is considered as an example.

This is an Open Access article distributed under the terms of the Creative Commons Attribution License 2.0, which permits unrestricted use, distribution, and reproduction in any medium, provided the original work is properly cited. 


\section{Composite-particle interaction in the framework of the RGM. One-channel problem}

The wave function of the resonating group model $[1,2]$ is chosen in the form

$$
\begin{aligned}
& \Psi_{A_{1}+A_{2}}=\hat{A}\left\{\Psi_{A_{1}} \Psi_{A_{2}} \Phi(\boldsymbol{\rho})\right\}, \\
& \hat{A}=\left(\begin{array}{l}
A \\
A_{1}
\end{array}\right)^{-1 / 2}\left(1+\sum_{p}(-1)^{p} \hat{P}\right),
\end{aligned}
$$

where the sum is over all permutations $\hat{P}$ of the $A$ nucleons, $p$ is the parity of a permutation and $\Phi(\boldsymbol{\rho})$ is a probe wave function. Inserting (1) to the $A$-fermion ( $\left.A=A_{1}+A_{2}\right)$ Schrödinger equation

$\hat{H}_{A} \Psi_{A}=E \Psi_{A}$,

$\hat{H}_{A}=\hat{T}+\hat{V}, \quad \hat{T}=\sum_{i=1}^{A} \frac{\hat{\mathbf{p}}_{i}^{2}}{2 m}$

$\hat{V}=\sum_{i<j=1}^{A} V\left(\mathbf{r}_{i}-\mathbf{r}_{j}\right)$

one can obtain two-body equation

$\left(\hat{T}_{\rho}+\hat{V}_{\rho}-E^{\prime} \hat{N}_{\rho}\right) \Phi(\boldsymbol{\rho})=0$

where

$E^{\prime}=E-E_{1}-E_{2}$,

and the normalization condition

$$
\begin{aligned}
& \left\langle\hat{N}_{\rho}^{1 / 2} \Phi(\boldsymbol{\rho}) \mid \hat{N}_{\rho}^{1 / 2} \Phi(\boldsymbol{\rho})\right\rangle= \\
& \left(1, \delta\left(E-E^{\prime}\right), \delta\left(k-k^{\prime}\right), \text { etc. }\right)
\end{aligned}
$$

for bound and continuous states, respectively. For a fixed value of the angular momentum $l$ of the relative motion the integral operators can be presented as

$$
\begin{aligned}
& \left(\begin{array}{c}
\hat{N}_{\rho, l} \\
\hat{T}_{\rho, l} \\
\hat{V}_{\rho, l}
\end{array}\right) \varphi_{l}(\rho) \equiv \int\left(\begin{array}{c}
N_{l}\left(\rho^{\prime}, \rho\right) \\
T_{l}\left(\rho^{\prime}, \rho\right) \\
V_{l}\left(\rho^{\prime}, \rho\right)
\end{array}\right) \varphi_{l}\left(\rho^{\prime}\right) \rho^{\prime 2} d \rho^{\prime}, \\
& \left(\begin{array}{c}
N_{l}\left(\rho^{\prime}, \rho^{\prime \prime}\right) \\
T_{l}\left(\rho^{\prime}, \rho^{\prime \prime}\right) \\
V_{l}\left(\rho^{\prime}, \rho^{\prime \prime}\right)
\end{array}\right)=\left\langle\hat{A}\left\{\Psi_{A_{1}} \Psi_{A_{2}} \frac{1}{\rho^{2}} \delta\left(\rho-\rho^{\prime}\right) Y_{l m}\left(\Omega_{\rho}\right)\right\}\right| \times \\
& \left(\begin{array}{c}
\hat{1} \\
\hat{T} \\
\hat{V}
\end{array}\right)\left|\hat{A}\left\{\Psi_{A_{1}} \Psi_{A_{2}} \frac{1}{\rho^{2}} \delta\left(\rho-\rho^{\prime \prime}\right) Y_{l m}\left(\Omega_{\rho}\right)\right\}\right\rangle .
\end{aligned}
$$

By action of the operator $\hat{N}_{\rho, l}^{-1}$ equation (6) can be transformed to a Schrödinger-like form

$$
\left(\hat{N}_{\rho, l}^{-1} \hat{T}_{\rho, l}+\hat{N}_{\rho, l}^{-1} \hat{V}_{\rho, l}-E^{\prime}\right) \varphi_{l}(\rho)=0 .
$$

However, the resulting Hamiltonian turns out to be nonHermitian one. Introducing a new wave function

$$
\phi_{l}(\rho)=\hat{N}_{\rho, l}^{1 / 2} \varphi_{l}(\rho)
$$

one can obtain the Schrödinger-like equation with Hermitian Hamiltonian

$$
\left(\hat{N}_{\rho, l}^{-1 / 2} \hat{T}_{\rho} \hat{N}_{\rho, l}^{-1 / 2}+\hat{N}_{\rho, l}^{-1 / 2} \hat{V}_{\rho, l} \hat{N}_{\rho, l}^{-1 / 2}-E^{\prime}\right) \phi_{l}(\rho)=0
$$

and usual normalization conditions

$$
\left\langle\phi_{E, l}(\rho) \mid \phi_{E^{\prime}, l}(\rho)\right\rangle=1
$$

for the states of discrete spectra, and

$$
\left\langle\phi_{E, l}(\rho) \mid \phi_{E^{\prime}, l}(\rho)\right\rangle=\delta\left(E-E^{\prime}\right)
$$

or similar - for the states in continuum.

In the RGM the functions $\Psi_{A_{1}}$ and $\Psi_{A_{2}}$ are most often considered as ground state oscillator wave functions (i. e. the lowest, compatible with the Pauli exclusion principle) with one and the same parameter $\hbar \omega$. In the algebraic version of RGM the function of the relative motion is sought in the form of the expansion

$$
\varphi_{l}(\rho)=\sum_{n} C_{n l} \phi_{n l}(\rho)
$$

over the oscillator basis (also characterized by the same parameter $\hbar \omega)$. Under these conditions the wave functions $\phi_{n l}(\rho)$ are eigenfunctions of the norm kernel

$$
\hat{N}_{\rho, l} \phi_{n l}(\rho)=\varepsilon_{n} \phi_{n l}(\rho)
$$

The eigenvalues $\varepsilon_{n}$ are equal to zero for forbidden states, and tend to unity as $n \rightarrow \infty$. The semi-forbidden states are defined as states with $\varepsilon_{n}$ considerably lower than unity. There are rare cases in which the eigenvalues are greater than unity. The mathematical formalism described below also allows one to include this states in the similar way as semi-forbidden states, thus we do not discuss this case separately.

Fundamental advantage of the algebraic version of OCM is a possibility to apply the following relation presented in Ref. [8]

$$
\left\langle\phi_{n l}\left|\hat{N}_{\rho, l}^{-1 / 2} \hat{T}_{\rho} \hat{N}_{\rho, l}^{-1 / 2}\right| \phi_{n^{\prime} l}\right\rangle=\sqrt{\frac{\varepsilon_{n_{<}}}{\varepsilon_{n_{>}}}} T_{n n^{\prime}}
$$

where $n_{<}=\min \left(n, n^{\prime}\right), \quad n_{>}=\max \left(n, n^{\prime}\right)$ and $T_{n n^{\prime}}$ is the matrix element of ordinary two-body kinetic energy operator between the oscillator functions. Due to that the 
set of the AV RGM equations looks as follows $\sum_{n^{\prime}}\left[\sqrt{\frac{\varepsilon_{n_{<}}}{\varepsilon_{n_{>}}}} T_{n n^{\prime}}+\left(\hat{N}_{\rho, l}^{-1 / 2} \hat{V}_{\rho, l} \hat{N}_{\rho, l}^{-1 / 2}\right)_{n n^{\prime}}-E^{\prime} \delta_{n n^{\prime}}\right] C_{n^{\prime} l}=0 ; n \geq n_{\min }$

so $A$-fermion exchange properties of the kinetic energy operator are completely determined by the norm kernel.

The basic approximation of the original OCM [4] is

$V\left(\rho^{\prime}, \rho^{\prime \prime}\right)=V\left(\rho^{\prime}\right) \delta\left(\rho^{\prime}-\rho^{\prime \prime}\right)$.

If the forbidden components are extracted from the function sought, then the initial equation becomes

$$
\left(\hat{T}_{\rho}+\hat{V}(\rho)-E^{\prime}\right) \tilde{\Phi}(\boldsymbol{\rho})=0
$$

where $\hat{V}(\rho)-$ direct (double folding) potential. Usually the exchange terms are neglected in the kinetic energy operator. In this approximation

$\hat{T}_{\rho}=\frac{\hat{\mathbf{p}}_{\rho}^{2}}{2 \mu}$.

In some cases the quality of description of data is not high enough in the initial variant of OCM. Other versions of OCM (presented in [9, 10] for example) utilize the phenomenological local potential in contrast to direct one and explore two alternative ways to take into account the Pauli exclusion principle:

1. Two-body model with forbidden states which are eigenstates of the Hamiltonian. This variant is rather simple because the redundant states are easily excluded due to their orthogonality to others in this case.

2. Two-body model with forbidden states which are eigenstates of the norm kernel $\hat{N}_{\rho}$

A possibility of adjusting of two-body local potential makes the approaches more flexible and improves the quality of the results.

The approach developed in the present paper allows one, first, to take into consideration the exchange terms of the kinetic energy completely and to use the second (well grounded microscopically) alternative way of exclusion of the forbidden states.

To demonstrate the formalism of the approach let us return to the expression (19). It should be noted that due to the equality (18) the potential energy term $\hat{V}_{\rho, l}$ in (13) remains the only term of the Hamiltonian in the AV RGM equations that includes the fermion exchange operators in the explicit form. It is just the term which is responsible for that RGM turns out to be a non-universal and overcomplicated model. The idea is to consider it phenomenologically approximating by a local potential form $\hat{V}_{c l}(\rho)$. After that the equation

$$
\left(\hat{\tilde{T}}_{\rho}+\hat{V}_{c l}(\rho)-E^{\prime}\right) \tilde{\Phi}(\boldsymbol{\rho})=0
$$

appears. The approach is called AV OCM independently of methods (which may be algebraic or that of "continuous" mathematics) applied to solve it.

In the algebraic versions of canonic two-body problem, RGM, or presented here OCM the expansion coefficients $C_{n l}$ (16) satisfy the infinite set of linear equations

$\sum_{n=0}^{\infty}\left(\left\langle\phi_{n^{\prime} l m}|\hat{H}| \phi_{n l m}\right\rangle-E \delta_{n^{\prime} n}\right) C_{n l}=0, n^{\prime}=0,1, \ldots$

which follows from the respective Schrödinger equation. For an ordinary bound states the eigenvalue problem

$\operatorname{det}\|\hat{H}-E \hat{I}\|=0$

is solved on the truncated basis $n \leq n_{\max }$. Here truncation means the boundary condition $C_{n l}=0, n>n_{\max }$ in the oscillator representation. For states of continuous spectra (including rather broad near-barrier resonances) the convergence of the functional series (16) is not uniform therefore so-called $J$-matrix method [11] is applied. The expansion coefficients decrease rather slowly as $n$ increase, and their asymptotic behavior should be introduced in the set of equations

$\sum_{n=0}^{N-1}\left(\left\langle\phi_{n^{\prime} l m}|\hat{H}| \phi_{n l m}\right\rangle-E \delta_{n^{\prime} n}\right) C_{n l}=-\sum_{n=N}^{\infty}\left\langle\phi_{n^{\prime} l m}|\hat{H}| \phi_{n l m}\right\rangle C_{n l}^{(a s)}$,

$n^{\prime}=0,1, \ldots$

The papers [5 - 7] were the first works in which the discussed method is applied to solve the RGM equations.

A high-precision form of the asymptotic coefficients is obtained in the papers [12-14]. In particular, for the wave function, asymptotically behave as the outgoing Coulomb wave, the expansion coefficient has the following form:

$$
\begin{aligned}
& C_{n l}^{(a s)}=\frac{1}{\sqrt{k r_{0} \varsigma_{n}}}\left\{G_{l}\left(\eta, k r_{0} \varsigma_{n}\right)+i F_{l}\left(\eta, k r_{0} \varsigma_{n}\right)-\right. \\
& \left.\frac{k^{3} r_{0}^{3}}{6 \varsigma_{n}}\left[G_{l}^{\prime}\left(\eta, k r_{0} \varsigma_{n}\right)+i F_{l}^{\prime}\left(\eta, k r_{0} \varsigma_{n}\right)\right]\right\}, \text { as } n \rightarrow \infty,
\end{aligned}
$$

where $r_{0}$ is the oscillator radius, $\varsigma_{n}=\sqrt{2 n+3}$, the prime denotes the derivatives of the Coulomb wave functions with respect to the second argument. The first term in the figure brackets in Eq.(27) provides a rather good approximation in the most cases.

Related approach may be useful also for calculations of the near-threshold bound states.

The presence of the forbidden states in (17) restricts the set of equations (26) and the sums in the left-hand side of them by the conditions $n, n^{\prime} \geq n_{\min }$. Semiforbidden states are taken into account by means of renormalization of the kinetic energy matrix. The explicit form of the of the kinetic energy matrix elements (18) is applied for that. In the oscillator basis the matrix of the 
renormalized kinetic energy operator (18) retains threediagonal form as the initial one.

In some cases it is hard to explore direct algebraic approach presented above because the asymptotic behavior of the expansion coefficients sought is achieved at too large distances and a huge basis in (16) is required. It is the case for a narrow resonance in a system decaying in two charged-composite-particle channel. In these situations it occurs more convenient to apply the methods of "continuous" mathematics. To do this a number of the separable terms related to the forbidden and the semiforbidden states are introduced into the Hamiltonian. The idea of this rearrangement is that the initial cluster Hamiltonian matrix elements $H_{n n^{\prime} ; l}$ between the states at least one of which is forbidden turn out to be cancelled with corresponding matrix elements of the separable terms. The matrix elements of the kinetic energy in the Hamiltonian $H_{n n^{\prime} ; l}$ are renormalized according to the formula (18) to account for the presence of semiforbidden states. The additional potential term which is denoted as $\hat{V}_{l}^{\text {sep }}$ takes the form

$\hat{V}_{l}^{\text {sep }}=-\sum_{n, n^{\prime}=0}^{n_{0}}|n l\rangle H_{n n^{\prime} ; l}\left\langle n^{\prime} l\right|-$

$\sum_{n=0}^{n_{0}} \sum_{n^{\prime}=n_{0}+1}^{\infty}\left(|n l\rangle H_{n n^{\prime} ; l}\left\langle n^{\prime} l|+| n^{\prime} l\right\rangle H_{n^{\prime} n ; l}\langle n l|\right)+$

$\sum_{n=n_{0}+1}^{\infty}\left(\sqrt{\frac{\varepsilon_{n}}{\varepsilon_{n+1}}}-1\right)\left(|n l\rangle T_{n, n+1 ; l}\langle n+1, l|+| n+1, l\rangle T_{n+1, n ; l}\langle n l|\right)$,

where

$\hat{H}=\hat{T}_{\rho}+\hat{V}_{c l}(\rho)$

Usually $\varepsilon_{n} / \varepsilon_{n+1}$ tends to unity rather rapidly as $n-$ increase (for example $\varepsilon_{n=80}=0.999$ for the system ${ }^{16} \mathrm{O}+$ ${ }^{16} \mathrm{O}$ ), therefore the sum in the $\hat{V}_{l}^{\text {sep }}$ can be truncated by relatively small value $n_{0}$.

Here we demonstrate the appropriate method to solve the Schrödinger equation

$\left(\frac{d^{2}}{d r^{2}}+k^{2}-2 V_{c l ; l}(\rho)\right) \chi_{l}(\rho)=2 \hat{V}_{l}^{s e p} \chi_{l}(\rho)$

with the additional separable terms. The solution of this equation $\chi_{l}(\rho)$ must behave asymptotically as $G_{l}(\eta, k \rho)+i F_{l}(\eta, k \rho)$ at large distances. The value of $G_{l}(\eta, k \rho)$ exceeds significantly the value of $F_{l}(\eta, k \rho)$ far left from the external turning point in the underbarrier domain. Thus according to the Ref. [15], it is enough to satisfy the matching condition with the function $G_{l}(\eta, k \rho)$ in this region only. Consequently solution $\chi_{l}(\rho)$ should satisfy the boundary conditions (i) $\chi_{l}(\rho)$ is regular at the origin $(\rho=0)$;

(ii) $\chi_{l}(\rho)$ behaves as $G_{l}(\eta, k \rho)$ under the barrier beyond the radius of the strong interaction.

To find the solution of the equation (30) let us consider the equation

$\left(\frac{d^{2}}{d r^{2}}+k^{2}-2 V_{c l ; l}(\rho)\right) \chi_{l}(\rho)=0$

with a local potential $V_{c l i l}(\rho)$ including the centrifugal part and introduce it's solutions: $\chi_{1 ; l}(\rho)$, which satisfies the condition (i); and $\chi_{2 ; l}(\rho)$ which satisfies (ii). The corresponding Green's function takes the form

$G\left(\rho, \rho^{\prime}\right)=\frac{\chi_{1 ; l}\left(\rho_{<}\right) \chi_{2 ; l}\left(\rho_{>}\right)}{W}$

where $\rho_{<}=\min \left(\rho, \rho^{\prime}\right), \quad \rho_{>}=\max \left(\rho, \rho^{\prime}\right) \quad$ and the Wronskian $W$ is written as follows:

$W=\chi_{1 ; l}(\rho) \frac{d \chi_{2 ; l}(\rho)}{d \rho}-\frac{d \chi_{1 ; l}(\rho)}{d \rho} \chi_{2 ; l}(\rho)$.

This Green's function allows one to deduce the homogeneous integral equation

$$
\chi_{l}(\rho)=-2 \int_{0}^{\infty} G\left(\rho, \rho^{\prime}\right)\left[\hat{V}_{l}^{s e p} \chi_{l}\right]\left(\rho^{\prime}\right) d \rho^{\prime}
$$

for the resonance solution $\chi_{l}(\rho)$. Here the notation $\left[\hat{V}_{l}^{\text {sep }} \chi_{l}\right]\left(\rho^{\prime}\right)$ means the function (depending on $\left.\rho^{\prime}\right)$ result of action of the operator $\hat{V}_{l}^{\text {sep }}$ on the function $\chi_{l}(\rho)$. The homogeneous equation (34) may have a solution only for the unique resonance energy values. Substituting the explicit form (28) of the potential $\hat{V}_{l}^{\text {sep }}$ into (34) one can obtain

$$
\begin{aligned}
& \chi_{l}(\rho)=2 \sum_{n=0}^{n_{0}} \int_{0}^{\infty} G\left(\rho, \rho^{\prime}\right) \phi_{n l}\left(\rho^{\prime}\right)\left\langle\phi_{n l}\left|V_{c l ; l}\right| \chi_{l}\right\rangle d \rho^{\prime}+ \\
& 2\left[\int_{0}^{\infty} d \rho^{\prime} G\left(\rho, \rho^{\prime}\right) \phi_{n_{0} l}\left(\rho^{\prime}\right) T_{n_{0}, n_{0}+1 ; l}+\right. \\
& \left.\int_{0}^{\infty} d \rho^{\prime} G\left(\rho, \rho^{\prime}\right) \phi_{n_{0}+2, l}\left(\rho^{\prime}\right)\left(1-\sqrt{\frac{\varepsilon_{n_{0}+1}}{\varepsilon_{n_{0}+2}}}\right) T_{n_{0}+1, n_{0}+2 ; l}\right]\left\langle\phi_{n_{0}+1, l} \mid \chi_{l}\right\rangle+ \\
& 2 \sum_{n=n_{0}+2}^{n_{\max }-1}\left[\int_{0}^{\infty} d \rho^{\prime} G\left(\rho, \rho^{\prime}\right) \phi_{n-1, l}\left(\rho^{\prime}\right)\left(1-\sqrt{\frac{\varepsilon_{n-1}}{\varepsilon_{n}}}\right) T_{n-1, n ; l}+\right. \\
& \left.\int_{0}^{\infty} d \rho^{\prime} G\left(\rho, \rho^{\prime}\right) \phi_{n+1, l}\left(\rho^{\prime}\right)\left(1-\sqrt{\frac{\varepsilon_{n}}{\varepsilon_{n+1}}}\right) T_{n, n+1 ; l}\right]\left\langle\phi_{n l} \mid \chi_{l}\right\rangle+
\end{aligned}
$$




$$
\begin{aligned}
& 2 \int_{0}^{\infty} d \rho^{\prime} G\left(\rho, \rho^{\prime}\right) \phi_{n_{\max }-1, l}\left(\rho^{\prime}\right)\left(1-\sqrt{\frac{\varepsilon_{n_{\max }-1}}{\varepsilon_{n_{\max }}}}\right) T_{n_{\max }-1, n_{\max } ; l} \times \\
& \left\langle\phi_{n_{\max }, l} \mid \chi_{l}\right\rangle,
\end{aligned}
$$

where $n_{\max }$ means the maximum value of the radial quantum number of truncated oscillator basis. The simple form of the first term is caused by the property of completeness of the oscillator basis allowing one to calculate the infinite sum over $n^{\prime}$ in the expression (28) explicitly.

There is an opportunity to treat Eq. (35) in the following way. Multiplying it by $\left\langle\phi_{n l}\right|$ and $\left\langle\phi_{n l}\right| V_{c l ; l}$ from the left-hand side one can obtain the set of homogeneous algebraic equations for the unknown coefficients $\left\langle\phi_{n l} \mid \chi_{l}\right\rangle$ and $\left\langle\phi_{n l}\left|V_{c l ; l}\right| \chi_{l}\right\rangle$. The condition of solvability (zero value of the determinant) determines the value $E_{\text {res }}$, after that the coefficients $\left\langle\phi_{n l} \mid \chi_{l}\right\rangle$ and $\left\langle\phi_{n l}\left|V_{c l i l}\right| \chi_{l}\right\rangle$ can be calculated. This procedure determines the function $\chi_{l}(\rho)$ and thus the width of the resonance.

However such a method of numerical calculations of the widths turns out to be unstable at least for narrow resonances in systems possessing a number of semiforbidden states with the eigenvalues of the norm kernel $\varepsilon_{n}$ strongly different from unity. In particular, very high accuracy (ten digits for $2 \mathrm{MeV}$ resonance state in ${ }^{16} \mathrm{O}+$ ${ }^{16} \mathrm{O}$ system) of the value $E_{\text {res }}$ is required to calculate the width reliably.

The way to overcome this difficulty looks as follows. Consider the above mentioned function obtained via direct algebraic approach

$$
\tilde{\chi}_{l}(\rho)=\sum_{n=n_{0}+1}^{n_{\max }} C_{n l} \phi_{n l}(\rho) \text {. }
$$

This function is a partial sum of the oscillator expansion of $\chi_{l}(\rho)$. It reproduces precisely the behavior of the wave function $\chi_{l}(\rho)$ in the interior domain. It is just that needs to calculate the values $\left\langle\phi_{n l} \mid \chi_{l}\right\rangle$ and $\left\langle\phi_{n l}\left|V_{c l i l}\right| \chi_{l}\right\rangle$, due to rapid decreasing of the functions $\phi_{n l}(\rho), \tilde{\chi}_{l}(\rho)$, and $V_{c l ; l}$ as $\rho$-increasing. Thus, substituting $\tilde{\chi}_{l}(\rho)$ into the right-hand side of the basic equation (35) one can obtain the solution for all the values of $\rho$, including the asymptotic region. Numerical calculations by means of the proposed method occur significantly more stable.

The width of a narrow resonance can be deduced from the solution $\chi_{l}(\rho)$ by means of the approach, presented in the monograph [15]. According to the approach the following asymptotic relation

$\chi_{l}(\rho) \approx \sqrt{\frac{\Gamma k}{2 E_{\text {res }}}} G_{l}(\eta, k \rho)$ is valid in the case that $G_{l}(\eta, k \rho) \gg>F_{l}(\eta, k \rho)$ in the under-barrier region far left from the external turning point. The normalization condition

$\int_{0}^{R} \chi_{l}^{2}(\rho) d \rho=1$

in the interior region is implied. The described above method of calculation of the resonance wave function with the aid of the equation (35) allows one to apply the formula (37) for determining of the decay width directly.

\section{Width of the lowest ${ }^{16} \mathrm{O}+{ }^{16} \mathrm{O}$ resonance state}

To demonstrate the effect of the forbidden and the semiforbidden states on the decay widths of nuclear states in the case of emission of heavy clusters let us consider the pair ${ }^{16} \mathrm{O}+{ }^{16} \mathrm{O}$ as an example. Three alternatives are studied: the OCM with the forbidden states considered as eigenstates of the two-body Hamiltonian - I, the OCM with the forbidden states belonging to the eigenstates of the norm kernel - II, and the OCM with the forbidden states of the latter type and the semi-forbidden states III. The local cluster-cluster potential of the squared Woods-Saxon form from [16]

$$
V_{c l}(\rho)=V_{\text {Coul }}(\rho)+\frac{V_{0}}{(1+\exp [(\rho-R) / a])^{2}}
$$

is considered. The Coulomb part is chosen in the form of interaction potential of the two uniformly charged spherical volumes. According to one of the versions of the model used in [16] there are 12 forbidden states (eigenstates of the Hamiltonian with the interaction (39)) and the narrow resonance state located at the energy $E=2.103 \mathrm{MeV}$ in the partial wave with $l=0$. The result is reproduced in our calculations realizing alternative I. For the alternatives II and III the depth $V_{0}$ of local potential $V_{c l}(\rho)$ is varied to restore the resonance energy of the alternative I. The values of the decay width for three versions of the OCM are presented in the Table 1. The resonance energy is presented to demonstrate the accuracy of its reproduction.

It is clear from the table, that the forbidden and notably semi-forbidden states change dramatically the decay width. If the forbidden states are considered as eigenstates of the norm kernel, then the value of the width $\Gamma$ becomes one order of magnitude smaller than the one, obtained assuming these states to be the eigenstates of the two-body Hamiltonian. If, in addition, the semi-forbidden states are also considered then the value of the width $\Gamma$ turns out to be eight orders of magnitude smaller.

Thus a very pronounced exchange effect displays in the properties of a resonance decaying into the channel with a long list of the semi-forbidden states which are significantly different from unity. 
Table 1. Width of the lowest ${ }^{16} \mathrm{O}+{ }^{16} \mathrm{O}$ resonance state for three versions of the interaction (see the text).

\begin{tabular}{|c|c|c|c|}
\hline Alternative & I & II & III \\
\hline$V_{0}, \mathrm{MeV}$ & 399.2 & 225.6 & 422.8 \\
\hline$E_{\text {res }}, \mathrm{MeV}$ & 2.103 & 2.103 & 2.102 \\
\hline$\Gamma, \mathrm{MeV}$ & $0.59 \cdot 10^{-27}$ & $0.53 \cdot 10^{-28}$ & $0.64 \cdot 10^{-35}$ \\
\hline
\end{tabular}

It should be noted that the parameters of the ${ }^{16} \mathrm{O}+{ }^{16} \mathrm{O}$ channel (the penetrability of the barrier, the eigenvalues of the norm kernel for the semi-forbidden states, the $\Gamma$ values) are more or less close to the ones typical for the alpha-decay of heavy nuclei. Therefore one may expect similar exchange effects in the latter process.

\section{Summary}

In the present paper a new version of the orthogonality conditions model is proposed. The model allows one to take into account exchange effects originated by the norm and the kinetic-energy overlap kernels. Both continuous and pure algebraic formalisms of the model are developed. The former one is used for the calculation of the decay widths of very narrow resonances. The example of such a resonance in ${ }^{16} \mathrm{O}+{ }^{16} \mathrm{O}$ channel is studied.

The results of this study demonstrate that:

1 Properties of the interaction of composite particles are essentially different from the ones of the structureless particles.

2. The basic cause of the differences is the exchange effects manifest themselves via the forbidden and the semi-forbidden states.

3. Algebraic approaches are convenient tools to consider these effects.

4. The methods developed here to describe the composite particles interaction are suitable for the calculations of various observables of composite-particle collisions.

\section{References}

1. J.A. Wheeler, Phys. Rev. 52, 1083 (1937)

2. J.A. Wheeler, Phys. Rev. 52, 1107 (1937)

3. T. Fliessbasch, H.-J. Mang, Nucl. Phys. A 263, 75 (1976)

4. S. Saito, Progr. Theor. Phys. 41, 705 (1969)

5. G.F. Filippov and I.P. Okhrimenko, Yad. Fiz. 33, 932 (1980)

6. G.F. Filippov and I.P. Okhrimenko, Yad. Fiz. 32, 928 (1980)

7. I.P. Okhrimenko, Nucl. Phys. A 424, 121 (1984)

8. H. Horiuchi, Prog. Theor. Phys., Suppl. 62, 90 (1977)
9. V.G. Neudatchin., A.N. Boyarkina., V.P. Korennoy et al., Nuovo Chim. Lett., 5, 834 (1971)

10. V.G. Neudatchin, V.I. Kukulin, V.P. Korennoy et al., Phys. Lett. B 34, 581 (1971)

11. H.A. Yamani, L. Fishman, J. Math. Phys. 16, 410 (1975)

12. S.Yu. Igashov, in The J-Matrix Method. Developments and Applications, A.D. Alhaidari, E.J. Heller, H.A. Yamani, and M.S. Abdelmonem (eds.) (Springer, 2008 ), p. 49.

13. S.Yu. Igashov, Comp. Math. and Math. Phys. 43, 78 (2003)

14. S.Yu. Igashov, Bull. RAS, Physics 65, 104 (2001)

15. S.G. Kadmensky, V.I. Furman, Alpha-decay and related nuclear reactions (Energoatomizdat, 1985) (in Russian)

16. Y. Kondö, in Proceedings of Intl. Conference "Microscopic Cluster Models of Light Nuclei and Related Topics" (Yukawa Inst. Theor. Phys., 1992), p. 191. 